\title{
Vorläufige Mitteilungen über die Ergebnisse einer Forschungsreise nach Ostafrika. ${ }^{1}$ )
}

Von

Dr. R. Koch.

Im Dezember 1904 begab ich mich im Auftrage des Kaiserlichen Gouvernements nach Deutsch-Ostafrika, um über die Bekämpfung des in der Kolonie angeblich weitverbreiteten Küstenfiebers der Rinder Untersuchungen anzustellen. $\mathrm{Zu}$ diesem Zwecke war es zunächst notwendig, die eigentliche Ausbreitung dieser Seuche in einer zuverlässigen Weise festzustellen. Da bekanntlich Rinder aus seuchenfreien Gebieten, wenn sie an die verseuchte Küste gebracht werden, erkranken, während diejenigen, welche aus verseuchten Gebieten stammen, daselbst gesund bleiben, so wurde beschlossen, von den wichtigeren Stationen der Kolonie Rinder nach Daressalam bringen und auf stark infizierten Stellen weiden zu lassen, um sie so auf ihre Seuchenfestigkeit resp. Seuchenempfänglichkeit zu prüfen.

Wegen der großen Entfernungen, welche die Tiere bei ihrem Transport zurückzulegen hatten, konnten sie erst nach 2-3 Monaten an ihrem Bestimmungsort eintreffen, und ich benutzte diese Zeit zu Studien über Rekurrens. Es war nämlich schon seit längerer Zeit aufgefallen, daß fast alle Europäer, welche auf der von Daressalam ins Innere führenden Karawanenstraße reisten, an R k u rrens erkrankten. Diese Rekurrensfälle waren unzweifelhaft schon immer vorgekommen, früher aber für Malaria gehalten und erst seit etwa einem Jahre auf Grund des mikroskopischen Blutbefundes richtig diagnostiziert worden. Nachdem sich erst einmal die Aufmerksamkeit auf diese Krankheit gelenkt hatte, war ihre Häufigkeit erkannt, und man hatte Untersuchungen über ihre Entstehungsursachen begonnen, welche bald dahin führten, eine Zeckenart als verdächtig anzusehen. Ich verschaffte mir eine Anzahl dieser Zecken, und es gelang mir, in einigen derselben Spirochaeten nachzuweisen, welche mit den Spirochaeten der Rekurrens identisch zu sein schienen. Dieser Befund forderte natürlich dazu auf, die Sache an Ort und Stelle, also auf der verseuchten Karawanenstraße, weiter zu verfolgen, und so unternahm ich in Begleitung des Oberstabsarztes Dr. M e ix n e r eine Expedition, welche uns auf der Karawanenstraße zehn Tagemärsche weit bis Morogoro führte.

Bald nach unserem Eintreffen daselbst erhielten wir die telegraphische Meldung, daß südlich von Mpapua, im Rubehogebirge, pe stve rd ä c b tig e Erkrankungen vorgekommen seien, denen ein auffallendes Sterben unter den Ratten vorhergegangen war. Diese alarmierende Nachricht veranlaßte uns, dorthin zu gehen und zu sehen, ob es sich um einen wirklichen Pestausbruch handelte. Aber glücklicherweise konnten wir feststellen, daß kein einziger unzweifelhafter Pestfall vorgekommen war, und daß

1) Aus Deutsche Medizinische Wochenschrift, 1905, Nr. 47. 
das Rattensterben nicht auf Pest beruhte, sondern durch die Maden einer Dasselfliege bedingt wurde.

Ich besuchte dann das Land Uhehe, wo vor zwei Jahren einige unzweifelhafte Fälle von Pest konstatiert waren und in letzter Zeit verdächtiges Rattensterben beobachtet wurde. Auch hier ließ sich dieselbe Ursache für das Rattensterben nachweisen.

Schon auf dem Marsche ins Innere des Schutzgebietes mußte ich mehrfach $\mathrm{T}$ s e t s e g e g e nden passieren, ganz besonders war dies aber auf dem Rückwege der Fall, auf dem ich einen Abstecher ins Ulugurugebirge machte, um einen ziemlich bedeutenden Lepraherd zu untersuchen. Es war nun eigentlich nicht meine Absicht gewesen, mich auf dieser Expedition mit Tsetsestudien zu befassen, aber die Gelegenheit war doch zu günstig, und so wurde ich eigentlich ganz gegen meinen Willen in Untersuchungen über die Tsetsefliegen hineingezogen, welche viel Zeit beanspruchten, aber auch zu interessanten Resultaten führten.

Nach meiner Rückkehr zur Küste fand ich eine Anzahl von Rindern vor, die inzwischen angekommen und zum Teil schon an Küstenfieber und Texasfieber erkrankt waren. Hier bot sich mir eine vorzügliche Gelegenheit, meine früher in Rhodesia begonnenen Untersuchungen über die Entwicklungsgeschichte der Piroplasmen wieder aufzunehmen. In Rhodesia war ich infolge der ungünstigen klimatischen Bedingungen in dieser Beziehung trotz aller darauf verwendeten Mühe nicht weit gekommen und haite nur die ersten Andeutungen einer Weiterentwicklung dieser interessanten Parasiten in den Zecken gefunden. Jetzt stand mir ein reiches Material in einem für die Entwicklung der Zecken sowohl als auch der von ihnen beherbergten Parasiten sehr geeigneten Klima zu Gebote, und es zeigte sich hier wieder so recht, wieviel bei derartigen Forschungen von der richtigen Wahl des Ortes und der Zeit abhängt. Es gelang mir diesmal ohne Schwierigkeit, die Entwicklungsgeschichte der Piroplasmen ziemlich weit, d. h. bis zu den Formen, zu verfolgen, welche sie in den Eiern der Zecken annahm.

Daneben wurden die Studien über die Tsetsefliegen fortgesetzt und, da Daressalam

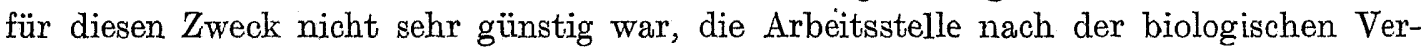
suchsstation Amani verlegt, wo ein gut eingerichtetes Laboratorium benutzt und die sich rings um das Usambaragebirge ausdehnenden Tsetseherde studiert werden konnten.

Da ich auch bei diesen Untersuchungen weitere nicht unwichtige Resultate erzielte, so hielt ich es für notwendig, bevor ich nach Europa zurückkehrte, Uganda zu besuchen, um mich durch eigene Anschauung über die Schlafkrankheit zu informieren und zu sehen, inwieweit meine Beobachtungen an den Glossinen der Tsetsekrankheit mit dem Verhalten der Glossina palpalis, der Überträgerin der Schlafkrankheit, übereinstimmten.

Von dem, was ich gelegentlich dieser in ihren Umrissen skizzierten Expeditionen beobachtet und entdeckt habe, will ich hier, vorbehaltlich einer späteren ausführlichen Darlegung, das Wichtigste in Kürze wiedergeben.

\section{Rekurrens.}

Die in Deutsch-Ostafrika vorkommende Rekurrenskrankheit weicht nur in wenigen Punkten von der europäischen Form dieser Krankheit ab. Besonders auffallend ist die Kürze der einzelnen Anfälle und die geringe Zahl der Spirochaeten im peripheren Blutstrom. Die afrikanischen Spirochaeten scheinen mir durchschnittlich länger zu sein als die europäischen. Ich habe niemals Chromatinkörper in den Spirillen gesehen, welche ihre Zugehörigkeit zu irgendwelchen Trypanosomen hätten vermuten lassen, auch keine Andeutungen von Flimmersaum, ebensowenig von Längsteilung. Dagegen bemerkt man 
an ihnen sehr häufig Lücken, weiche den Eindruck machen, als ob sie sich durch Querteilung (Fig. 1) ${ }^{1}$ ) vermehren.

Durch Einspritzung von Rekurrensblut unter die Haut von Affen können diese mit Sicherheit infiziert werden. Die künstlich erzeugte Krankheit verläuft bei den Affen meistens schwer, selbst tödlich. Im Blute finden sich die Spirochaeten reichlicher als beim Menschen²). Es ist nicht gelungen, Rinder, Hunde und andere Versuchstiere zu infizieren.

Die afrikanische Rekurrens wird durch den Stich einer Zecke übertragen ${ }^{3}$ ).

Diese Zecke, Ornithodorus moubata Murray, lebt im Boden der Eingeborenenhütten; sie kommt nachts heraus, saugt das Blut der Menschen und versteckt sich tagsüber wieder in der Erde. Man findet sie regelmäßig auch im Boden der sogenannten Banda, des Schutzdaches, unter dem die Karawanenleute übernachten, aber nur, soweit der Boden nicht vom Regen getroffen wird. Die Zecken sind wahrscheinlich über das ganze Schutzgebiet verbreitet, sie finden sich auch in Ortschaften, welche fern vom Karawanenverkehr liegen.

Wenn eine Zecke das Blut eines rekurrenskranken

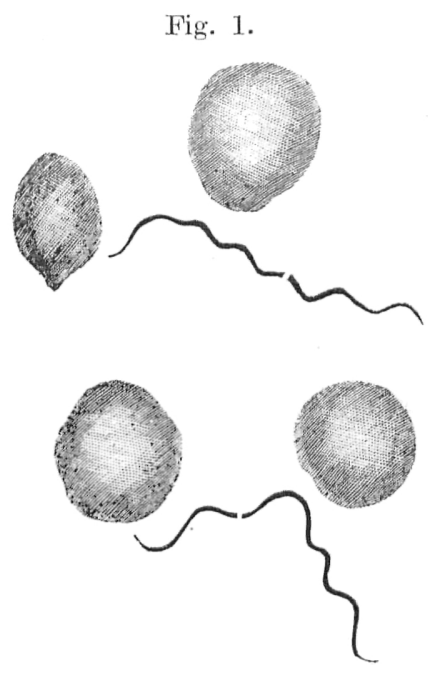

Menschen oder Affen gesogen hat, dann vermehren sich die Spirochaeten nicht, sondern sie verschwinden im Laufe von einigen Tagen aus dem Magen der Zecke. Untersucht man aber solche Zecken eingehender, dann findet man in einer gewissen Anzahl derselben die Spirochaeten an der Oberfläche des Ovarium. Diese Untersuchung hat in der Weise zu geschehen, daß man das Ovarium herauspräpariert, auf einem Deckglase zerquetscht, gut verteilt, trocknen läßt und mit Azur-Eosin-Mischung färbt. Meistens finden sich die Spirochaeten dann in solcher Zahl und Anordnung, daß eine erhebliche Vermehrung derselben stattgefunden haben muß. Am besten gelingt dieser Nachweis bei Ovarien mit unentwickelten Eiern.

Nachdem die Zecken ihre Eier abgelegt haben, lassen sich die Spirochaeten auch im Inhalte der Eier nachweisen. Es sind aber nur immer einzelne Gelege, und in diesen auch nur wieder einzelne Eier, welche mit Spirochaeten infiziert sind. Anfangs finden sich nur einzelne Spirochaeten oder Gruppen von wenigen Exemplaren in den Eiern, später sind sie zahlreicher und bilden öfters Haufen (Fig. 2.) Sie scheinen sich daher in den Eiern noch weiter zu vermehren. Irgendwelche Veränderungen, welche auf einen Entwicklungskreis der Spirochaeten schließen lassen könnten, habe ich nicht beobachtet.

Die aus infizierten Gelegen hervorgehenden jungen Zecken sind imstande, Affen, welchen sie angesetzt werden, mit Rekurrens zu infizieren $\left.{ }^{4}\right)$.

$\left.{ }^{1}\right)$ Die Holzschnitte sind nach Zeichnungen angefertigt, welche Oberarzt Dr. K u d i cke möglichst naturgetreu mit dem Z e i $B$ schen Zeichenapparat bei 2500 facher Vergrößerung nach den Originalpräparaten gemacht hat.

2) Erfolgreiche Übertragungen von afrikanischer Rekurrens auf Affen hat Oberarzt Dr. K u d i c k e bereits Ende 1903 ausgeführt.

3) Da mir während der Expedition die betreffende Literatur nicht zur Verfügung stand, so habe ich erst später erfahren, daß fast gleichzeitig mit mir der leider zu früh verstorbene, ausgezeichnete Forscher D u t to n Affen durch Zecken mit Rekurrens infiziert hat. Vgl. British Med. Journ. Febr. 4. 1905 und May 6. 1905.

4) Bis jetzt ist dieser Versuch elfmal erfolgreich ausgeführt. 
Unter den auf Spirochaeten untersuchten Zecken fanden sich gewöhnlich 5 bis 15 Proz. infizierte, in einzelnen Fällen stieg diese Zahl auf 50 Proz.

Infizierte Zecken wurden fast in allen Orten der Karawanenstraße von Daressalam bis über Kilossa hinaus in der Richtung nach Mpapua und auf der Strecke von Kilossa bis Iringa gefunden. Sie fehlten aber auch nicht in den Dörfern des Rubehogebirges und in Ortschaften, welche abseits vom Karawanenverkehr liegen.

Von den drei Europäern, welche sich bei der Karawane befanden, erkrankte keiner an Rekurrens, weil dieselben niemals in Hütten der Eingeborenen und nicht unter den Banden übernachteten. Von den fünf eingeborenen Dienern, welche in den Hütten oder in den Banden zu schlafen pflegten, erkrankten vier an Rekurrens. Von den 60 Trägern bekam keiner Rekurrens, obwohl sie auch in den Hütten und unter den Schutzdächern schliefen, offenbar weil sie bei früheren Expeditionen schon infiziert wurden und nach überstandener Krankheit immun geworden waren.

Fig. 2.
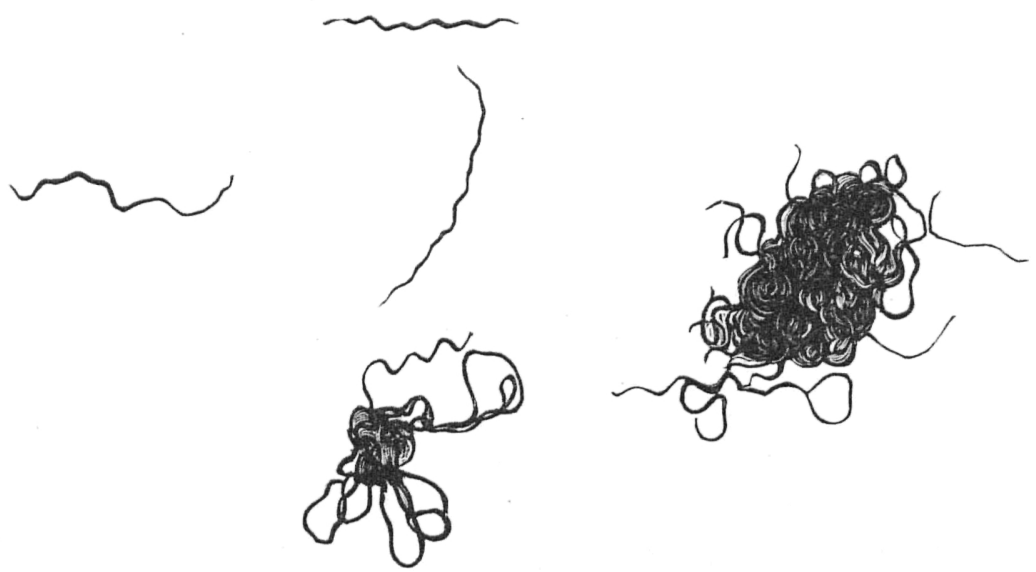

Gewisse Beobachtungen sprechen dafür, daß die Rekurrens in Deutsch-Ostafrika in weiter Verbreitung seit jeher endemisch herrscht, daß die Eingeborenen die Krankheit in der Regel in der Jugend überstehen und infolgedessen mehr oder weniger immun werden, so daß sie gar nicht mehr oder nur mit einmaligen leichten Anfällen erkranken. Der Europäer kann sich gegen die Infektion am einfachsten dadurch schützen, daß er sein Zelt an solchen Stellen aufschlägt, wo niemand vor ihm gelagert hat.

Zufälligerweise fand ich unter den in Daressalam untersuchten Rindern zwei, welche an der von Th e il e r entdeckten Spirillose der Rinder litten. Auch bei diesen Tieren konnte ich die Spirochaeten bis in die Eier der Zecken verfolgen.

2. Entwicklung des Piroplasma bigeminum.

Das Piroplasma bigeminum, der Erreger des Texasfiebers, kann unter bestimmten klimatischen Bedingungen und in gewissen Zecken einen eigentümlichen Entwicklungsgang durchmachen.

Um diese Entwicklung zu verfolgen, untersucht man den Mageninhalt der von einem infizierten Tiere abgenommenen voll Blut gesogenen Zecken an mehreren aufeinanderfolgenden Tagen.

Der birnförmige Parasit, dessen Chromatin sich in der Regel schon vorher in zwei voneinander getrennte Massen geteilt hat (Fig. 3), verläßt das rote Blutkörperchen und 
streckt sich in die Länge, wobei einer der beiden Chromatinkörper an das vordere Ende des Parasiten tritt und eine dunkel gefärbte scharfe Spitze bildet. Die andere Chromatinmasse bleibt ungefähr in der Mitte des Parasiten liegen; sie hat ein weniger kompaktes Aussehen (Fig. 4). Es erscheinen darauf strahlenartige Ausläufer unterhalb der

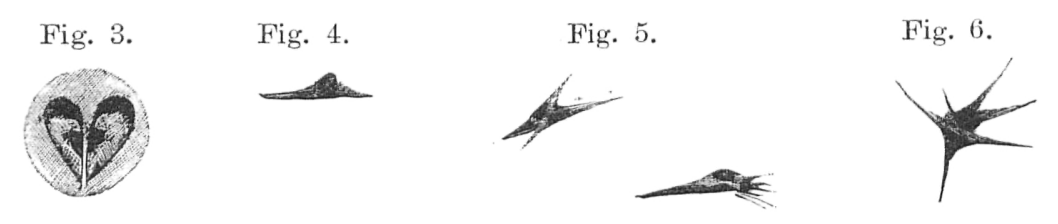

Spitze, anfangs zwei bis drei, später mehr. Auch am unteren Ende des Parasiten bilden sich oft mehrere Strahlen oder Zacken. Immer hat der Parasit ein eckiges, strahliges Aussehen (Fig. 5 und 6). Oft gleicht er einem unten spitz zulaufenden Kolben, an dessen oberem Ende ein von Strahlen umgebenes Chromatinkorn wie ein Stern sitzt (Fig. 7 und 8).

Vom zweiten Tage ab finden sich neben den eben beschriebenen Formen oft noch solche, bei denen zwei Individuen an ihren unteren Enden miteinander verbunden sind, so daß also ein Körper entsteht, der ein Mittelstück und an beiden Enden desselben

Fig. 7.

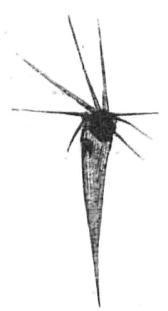

Fig. 8 .

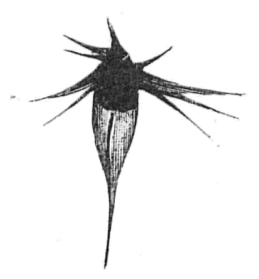

Fig. 9 .

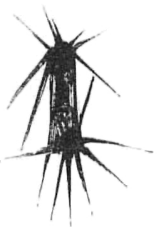

Fig. 10.

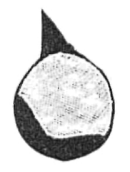

mit Strahlen besetzte, sternähnliche Chromatinkörner besitzt (Fig. 9). Ich möchte diese Form für eine Art von Kopulation halten. Dann treten kugelige Gebilde auf, deren innere Wand streckenweise mit Chromatin belegt ist und die an der Peripherie noch eine Chromatinspitze tragen (Fig. 10). Es hat den Anschein, als ob dieselben aus kopulierten Parasiten hervorgegangen sind, welche die strahligen Fortsätze abgeworfen haben.

Die mit Strahlen versehenen Parasiten haben eine große Neigung, sich zu Gruppen zu vereinigen. Man findet sie oft in Haufen von drei bis zu zehn und mehr Exemplaren, unter denen sich gewöhnlich auch kopulierte Paare befinden.

Ferner trifft man mitunter längliche, ovale oder birnförmige Körper, welche im blau gefärbten Plasma einen ziemlich großen Chromatinkern von körniger Beschaffenheit besitzen (Fig. 11). Diese letzteren scheinen mir den Übergang zu bilden zu den verhältnismäßig großen, ebenfalls birnförmigen Formen, welche ich öfters in den Eieren der infizierten Zecken angetroffen habe (Fig. 12 und 13). Sie Fig. 11. Fig. 12. Fig. 13. sind drei- bis viermal so groß als die Piroplasmen im Blute

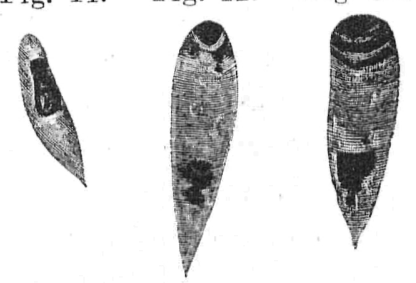
der Rinder, und es ist deswegen wohl anzunehmen, daß zwischen diesen beiden Formen noch Übergänge existieren. Dieselben werden in den jungen Zecken zu suchen sein, sei es noch innerhalb des Eies oder unmittelbar nach dem Ausschlüpfen, da bekanntlich 
die junge Zecke imstande ist, zu infizieren. Bis jetzt ist es mir nicht gelungen, diese Übergangsform zu finden.

Die Entwicklungszustände des Piroplasma bigeminum konnte ich im Rhipicephalus australis, Rhip. Evertsi und im Hyalomma aegyptium nachweisen, und zwar nur in ausgewachsenen und vollgesogenen Zecken und in deren Eiern. Sie fanden sich niemals bei den oft untersuchten Larven, Nymphen und nicht vollgesogenen geschlechtsreifen Exemplaren.

\section{Küstenfieber der Rinder.}

Die Parasiten des Küstenfiebers weichen in ihrem Verhalten in mehrfacher Beziehung von denjenigen der bekannten Piroplasmen vom Rind, Hund und Pferd so weit ab, daß es den Anschein hatte, als ob sie mit diesen nicht artverwandt seien.

Sie zeigen nicht die regelmäßige Zweiteilung, wie sie für die Piroplasmen so charakteristisch ist. Dagegen finden sich beim Küstenfieber regelmäßig vierfach geteilte Parasiten, welche in Kreuzform ( $: \cdot$ ) angeordnet sind. Letztere treten niemals bei den echten Piroplasmen auf; aber sie kommen auch bei der von Dschunkowski im transkaukasischen Rußland entdeckten, von ihm als tropische Piroplasmose benannten Krankheit vor ${ }^{1}$. Auch beim Pferde gibt es neben der echten Piroplasmosis eine Krankheit mit Parasiten in Kreuzform²). Es empfiehlt sich deswegen, diese Krankheiten, bei welchen die Parasiten in Kreuzfórm vorkommen, zu einer Gruppe zusammenzufassen.

Eine weitere Eigentümlichkeit des Küstenfiebers besteht darin, daß bei demselben in Milz und Lymphdrüsen kugelförmige Gebilde in großer Anzahl gefunden wurden, welche aus blau gefärbtem Plasma bestehen und in letzterem eine Anzahl Chromatinkörper enthalten (die nähere Beschreibung dieser Gebilde, deren Bedeutung noch nicht genügend aufgeklärt ist, muß ich mir für die ausführliche Arbeit vorbehalten. Dieselben sind schon vor dem Auftreten der Parasiten im Blute so regelmäßig in Milz und Drüsen nachzuweisen, daß ich sie zur frühen Diagnose der Krankheit bei geschlachteten Tieren mit Vorteil verwenden konnte.

Es ist mir nun gelungen, auch für die Parasiten des Küstenfiebers wenigstens die ersten Entwicklungsstufen, welche sie in der Zecke durchmachen, zu entdecken. Sie verhalten sich dabei ganz analog dem Piroplasma bigeminum, indem sie ebenfalls eckige, mit

Fig. 14.

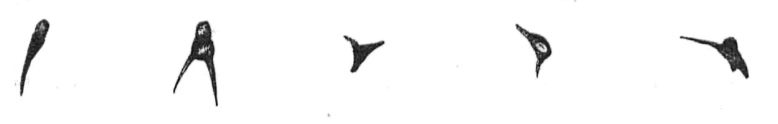

Strahlen versehene Formen annehmen. Nur sind dieselben erheblich kleiner und mit weniger Ecken und Strahlen versehen (Fig 14). Es folgt daraus, daß die Küstenfieberparasiten trotz der erwähnten Abweichungen entwicklungsgeschichtlich den echten Piroplasmen sehr nahestehen.

Zu erwähnen ist noch, daß die Entwicklung dieser Parasiten bisher nur in ausgewachsenen und vollgesogenen Exemplaren des Rhipicephalus australis beobachtet wurde, niemals in anderen Zeckenarten oder in jüngeren Stadien des Rhipicephalus australis. Dies würde dafür sprechen, daß beim Küstenfieber die Infektion nur durch die jungen

1) Durch den Nachweis dieser Kreuzform konnte ich die Verbreitung dieser dem Küstenfieber sehr nahestehenden Rinderkrankheit in den Mittelmeerländern, in Ostafrika und in Neu-Guinea feststellen.

2) Von Dr. K u d i c k e wurden derartige Parasiten auch bei einem Zebra nachgewiesen. 
Zecken dieser oder anderer Arten erfolgt, in welchen etwa später die Weiterentwicklung des Parasiten nachgewiesen werden sollte.

\section{Tsetsefliegen und Trypanosomen.}

Auf meinen Expeditionen habe ich vier verschiedene Arten der Gattung Glossina gefunden. Es sind Glossina fusca, morsitans, pallidipes und tachinoides. Dazu kommt die von Dr. F e l d m a $\mathrm{n} n$ auf den zum deutschen Gebiete gehörigen Inseln des VictoriaNjansa nachgewiesene Glossina palpälis und höchstwahrscheinlich noch Glossina longipennis, welche ich bei der Fahrt mit der Ugandaeisenbahn zwischen den Stationen Voi und Tsavo, nicht fern von der deutschen Grenze, gefangen habe. Somit kommen auf Deutsch-Ostafrika sechs von den acht bisher beschriebenen Glossinenarten.

Bisher nahm man an, daß Glossina morsitans die Überträgerin der Tsetsekrankheit sei. Diese Glossinenart findet sich aber nur strichweise im Schutzgebiet, besonders im Süden und im Norden zwischen den großen Seen. In der Mitte tritt an ihre Stelle Glossina pallidipes.

Glossina tachinoides, die kleinste der Glossinen, scheint weit verbreitet zu sein, tritt aber immer nur in wenigen Exemplaren auf. Dagegen bin ich der Glossina fusca überall, wo năchweislich die Tsetsekrankheit herrscht, in großer Zahl begegnet.

Bei der Gl. fusca ist es mir auch zuerst gelungen, mit Trypanosomen infizierte Exemplare zu entdecken, und zwar auf folgende Weise. Bei der Untersuchung der Fliegen unter dem Präpariermikroskop fiel es mir auf, daß der Stechrüssel regelmäßig mit Flüssigkeit gefüllt ist, welche durch Druck auf den Bulbus des Rüssels in Form eines wasserhellen Tröpfchens ausgepreßt werden kann. Da zu vermuten war, daß die Fliege beim Stechen dieses Tröpfchen unter die Haut ihres Opfers entleert, und daß infizierte Fliegen in der Flüssigkeit Trypanosomen enthalten müssen, so präparierte ich eine Anzahl von Rüsseln, untersuchte die ausgepreßten Tröpfchen mikroskopisch und fand in der Tat bei einigen Fliegen massenhaft Trypanosomen. Der Umstand, daß die Flüssigkeit ganz frei von roten Blutkörperchen war, daß die Trypanosomen viel zahlreicher waren, als sie jemals im Blute vorkommen, und daß sie verschiedene Entwicklungsstadien zeigten, ließ sofort erkennen, daß diese Fliegen nicht, wie bisher angenommen wurde, die Krankheit durch Überimpfung von Blut eines Kranken auf ein gesundes Tier direkt übertragen, sondern daß hier ähnliche Verhältnisse vorliegen wie bei den Malariaparasiten, daß die Trypanosomen also in der Glossina einen Entwicklungsgang durchmachen. Diese Annahme fand später ihre volle Bestätigung, als ich die Fliegen, deren Rüssel Trypanosomen enthielten, einer genauen Untersuchung unterwarf und fand, daß der Magen derselben in seinen verschiedenen Abschnitten, auch wenn er ganz frei von Blut war, große Mengen von Trypanosomen in verschiedenen Entwicklungszuständen enthielt. Im Darm fehlen sie oder sind nur in geringer Zahl vorhanden. In allen übrigen, namentlich auch in den drüsigen Organen Schläuchen habe ich niemals mit Sicherheit Trypanosomen nachweisen können:

Es wurden im ganzen nahezu 60 trypanosomenhaltige Fliegen untersucht, darunter auch eine Gl. morsitans und eine Gl. pallidipes.

Danach scheint es mir erwiesen, daß die Tsetsekrankheit im deutschen Schutzgebiete nicht allein durch die GI. morsitans übertragen wird, sondern auch durch die Gl. pallidipes und hauptsächlich durch Gl. fusca, was auch mit meinen Beobachtungen über das Vorkommen dieser verschiedenen Glossinenarten vollkommen übereinstimmt.

Der Entwicklungsgang der Trypanosomen in der Glossina gestaltet sich nach meinen Untersuchungen folgendermaßen: Die mit dem Blute aufgenommenen Trypanosomen, deren Zahl unter den natürlichen Verhältnissen wohl immer eine sehr beschränkte ist, 
vermehren sich im Magen durch Längsteilung sehr bald und nehmen auch an Größe erheblich zu, wie ein Vergleich zwischen Fig. 15, welche die Abbildung eines Trypanosoma aus dem Blute eines Rindes gibt, und Fig. 16, einem Trypanosoma aus dem Magen einer infizierten Glossina, lehrt. Zugleich tritt eine sehr auffallende Differenzierung ein. Ein Teil der großen Trypanosomen nimmt eine dicke, plumpe Gestalt an, ist reich an blau gefärbtem Plasma und hat einen ziemlich großen rundlichen Chromatinkörper von lockerem Gefüge (Fig. 16), während ein anderer Teil der Parasiten äußerst schlank aussieht, kein blau gefärbtes Plasma, aber einen langen dünnen, sehr dicht gefügten Chromatinkörper hat (Fig. 17). Bald ist der eine Typus überwiegend, bald der andere. Stellenweise kann man dichte Haufen des einen, an anderen Stellen desselben Präparates ein Gewimmel des anderen Typus treffen. Es verhält sich also

Fig. 16 .

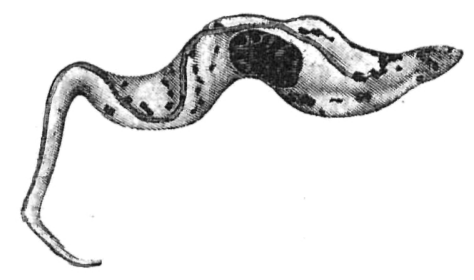

Fig. 17.

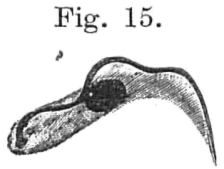

ähnlich wie bei den Malariaparasiten, die beim Beginn ihrer sexuellen Entwicklungsperiode eine Differenzierung in plasmaarme Formen mit reichlichem dichtem Chromatinkörper und in plasmareiche mit lockerem Chromatinkörper zeigen. Ich nehme deswegen an, daß es sich auch bei den Trypanosomen in diesem Stadium um die Bildung von sexuellen, d. h. männlichen und weiblichen Formen handelt, und daß die plumpen plasmareichen die weiblichen, die schlanken plasmaarmen die männlichen Trypanosomen $\operatorname{sind}^{1}$ ).

$\mathrm{Ob}$ nun weiter eine Kopulation oder, wie bei den Malariaparasiten, eine Bildung von Mikrogameten stattfindet, vermag ich noch nicht mit Sicherheit zu entscheiden. Doch sprechen einige Befunde dafür, daß bei den Trypanosomen der letztere Vorgang die Regel ist.

Weiter findet man in dem untersten Abschnitt des Magens Formen, welche mir die Weiterentwicklung der befruchteten Weibchen vorzustellen scheinen. Es sind sehr

Fig. 18.

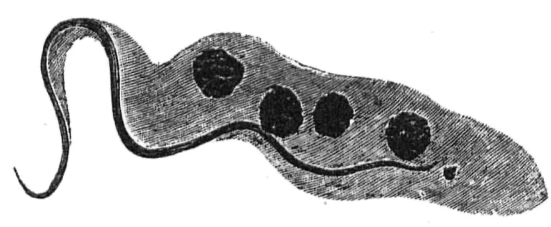

Fig. 19.

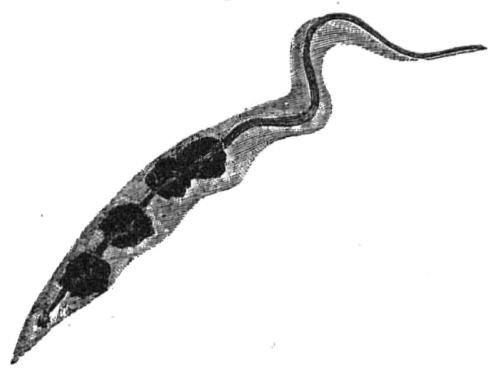

große Trypanosomen, welche nur einen Blepharoplasten mit zugehöriger Geißel, aber mehrere Kerne besitzen. Die Zahl der Kerne beträgt zwei, meistens vier (Fig. 18 und 19), in einigen Fällen auch acht.

$\left.{ }^{1}\right)$ Inwieweit diese sexuellen Formen von früheren Beobachtern gesehen sind, werde ich in der ausführlichen Arbeit erörtern. 
Eine Kernvermehrung tritt bekanntlich bei den Trypanosomen auch bei dem einfachen Teilungsvorgang ein, und es können dabei ebenfalls mehr als zwei Kerne in einem Individuum vorkommen, dann findet sich aber ausnahmslos eine ebenso große Zahl von Blepharoplasten und Geißeln wie Kerne.

Da bei den oben beschriebenen Formen nur die Kerne, aber nicht die Blepharoplasten vermehrt sind, so kann es sich nicht um eine einfache Teilung, sondern um einen andern Vermehrungsvorgang handeln, und ich halte es für sehr wahrscheinlich, daß, obwohl ich diesen Vorgang noch nicht beobachtet habe, diese vielkernigen Trypanosomen in entsprechend viele Teile zerfallen und damit die jüngsten Formen liefern, welche mir in den infizierten Fliegen oft begegnet sind ${ }^{1}$ ). Dieselben bilden einfache kuglige Zellen mit einem Kern (Fig. 20) und zeigen dann weiter alle Übergänge zu solchen mit Kern und Blepharoplast, zu welchem dann ein Geißelfaden tritt (Fig. 21 und 22); dann
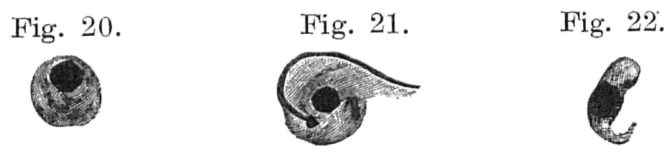

Fig. 23.

wird die Gestalt länglich und nimmt immer mehr die Trypanosomenform an. Gewöhnlich sind diese Jugendformen daran zu erkennen, daß der Blepharoplast vor dem Kern (in der Richtung nach der Geißel zu) liegt (Fig. 23); erst später tritt er neben den Kern und rückt nur ganz allmählich an das hintere Ende des Trypanosoma.

Neben diesen Formen treten aber gelegentlich noch andere auf, für welche ich bisher noch keine annehmbare Erklärung finden konnte. Es sind zu Längsbündeln

Fig. 24 .

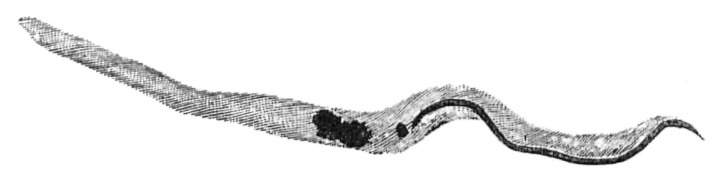

vereinigte lange dünne Trypanosomen, welche oft aufgerollt sind und dann so aussehen, als hätten sie sich aus einer großen Zelle durch Segmentierung des Inhalts entwickelt. Ferner schmale bandförmige Trypanosomen, oft von außergewöhnlicher Länge und mit abgestutzten Enden, in denen der Blepharoplast weit nach vorn gelagert ist und nur einen ganz kurzen Geißelfaden hat. (Fig. 24.)

$\mathrm{Zu}$ bemerken ist noch, daß in der Rüsselflüssigkeit neben den anderen Formen fast regelmäßig Trypanosomen gefunden werden, welche ganz das Aussehen und die Größe der Bluttrypanosomen besitzen. Es läßt sich wohl annehmen, daß durch diese die Infektion bewirkt wird. Der Versuch, Ratten mit den im Magen der Fliege enthaltenen Trypanosomen zu infizieren, blieb resultatlos ${ }^{2}$.

Bisher ist es auch nicht mit Sicherheit gelungen, Glossinen durch Fütterung an Tieren, welche Trypanosomen im Blute haben, zu infizieren. Wenn sie an Rindern gefüttert wurden, welche frisch an Tsetse erkrankt waren und viele Trypanosomen hatten,

1) Dieselben Jugendformen haben schon Rabinowits ch und Kempner bei den Ratten-Trypanosomen, ferner $\mathrm{N}$ ov y und $\mathrm{M}$ ' $\mathrm{N}$ e a 1 in ihren Trypanosomen-Kulturen, vielleicht auch $\mathrm{Cas}$ tellani in der Cerebrospinalflüssigkeit von Schlafkranken gesehen.

2) Zu demselben negativen Ergebnis ist die Sleeping Sickneß-Kommission bei ihren Versuchen gelangt, die Trypanosomen aus der Gl. palpalis auf Affen zu übertragen. Reports of the Sleep. Sickn. Commission of the Royal Society No. VI, p. 286. 
verschwanden die Trypanosomen in dem Maße, als das Blut verdaut wurde; aber es kam weder eine Entwicklung der Trypanosomen noch eine dauernde Infektion der Fliegen zustande. Erst als die Fliegen an Tieren (Ochsen, Maultieren) gefüttert wurden, welche vor langer Zeit infiziert waren und nur gelegentlich wenige Trypanosomen im Blute hatten, wurden einige Fliegen infiziert. Ich möchte daher annehmen, daß nicht alle Bluttrypanosomen zur Infektion der Glossinen geeignet sind, sondern nur solche, welche sich in einem bestimmten, uns noch nicht genügend bekannten Zustande befinden. Vermutlich finden sich die so beschaffenen Trypanosomen in den für Tsetse wenig empfänglichen Tieren, wie in den großen Wildarten (Antilopen, Büffel). Ich habe die infizierten Fliegen öfters in solchen Gegenden gefunden, wo sie sich nur am großen Wild infiziert haben konnten, in dessen Blut bekanntlich die Trypanosomen nur in höchst spärlicher Zahl vorhanden sind. Im Luengeratal, wo der höchste Prozentsatz von infizierten Fliegen gefunden wurde (17,4 Proz.), gab es keine Rinder, nur sehr wenige Antilopen, dagegen Ziegen und Schafe; die scheinbar ganz gesund waren, aber zum großen Teil vereinzelte Trypanosomen in ihrem Blute hatten.

In bezug auf das Verhalten der Glossinen ist noch bemerkenswert, daß sowohl Männchen als Weibchen Blut saugen und daß beide Geschlechter infiziert werden und demnach auch wieder infizieren können.

Die Glossina fusca fliegt und sticht auch zur Nachtzeit. Um sie in der Gefangenschaft längere Zeit am Leben zu erhalten, muß ihr alle zwei bis drei Tage Gelegenheit gegeben werden, Blut zu saugen. Die Männchen der Glossina fusca sitzen mit Vorliebe auf dem Boden und auf den Sträuchern und Büschen am Wege. Die Weibchen halten sich mehr versteckt und kommen erst zuim Vorschein, wenn sie Tiere wittern. Wenn man die Fliegen ohne Zuhilfenahme von Tieren fängt, bekommt man deswegen fast nur Männchen. Unter den Fliegen, welche an Tieren (Maultieren, Eseln, Rindern) gefangen werden, befinden sich stets auch Weibchen, mitunter ebensoviel wie Männchen.

Die Weibchen legen nicht Eier wie andere Dipteren, sondern immer nur eine einzelne weißliche Larve, welche sich nach wenigen Stunden verpuppt. Zwischen dem Ablegen der einzelnen Larven verstreicht bei der Glossina fusca, wenn sie regelmäßig gefüttert wird, je nach der Lufttemperatur, ein Zeitraum von 10-20 Tagen. Das Weibchen produziert also in einem Monat nicht mehr als zwei bis drei Nachkommen. Die Fortpflanzung der Glossinen geht also im Verhältnis zu anderen Insekten ungemein langsam vor sich. Hier scheint mir die schwächste Stelle in dem Infektionskreislauf der Trypanosomen zu liegen, welche vielleicht einen Anhaltspunkt für eine wirksame Bekämpfung der Trypanosomenkrankheiten bieten kann. 